\title{
The pea aphid genome sequence brings theories of insect defense into question
}

\author{
Christine G Elsik*
}

\section{Abstract \\ The genome sequence of the pea aphid is the first for a basal hemimetabolous insect and provides insights into developmental plasticity, symbiosis and insect immunity.}

\section{The pea aphid life cycle}

The ecology, physiology and evolution of the hemipteran insect pea aphid (Acyrthosiphon pisum) has been well studied because of its fascinating phenotypic plasticity, its heritable symbiotic associations and its impact on agriculture. Aphids are soft-bodied sap-feeding insects that act as vectors for plant viruses and cause worldwide crop damage. Sequencing and analysis of the pea aphid genome by the International Aphid Genomics Consortium (IAGC) [1] has provided new insights into aphid development and their interactions and coevolution with obligate and facultative symbiotic bacteria. Among the studies enabled by the genome project is the characterization of genes involved in the pea aphid immune and defense systems, published in this issue of Genome Biology [2].

The genome of the pea aphid is the first to be sequenced of the hemimetabolous group of insects, characterized by life cycles with incomplete metamorphosis from juvenile to adult stages. The annual aphid life cycle is particularly interesting because it includes a single sexual generation that alternates with several consecutive all-female parthenogenic generations (reviewed in [3]). The sexual males and females mate in the autumn, producing diapausing eggs that overwinter and hatch in the spring to produce the first all-female generation. The reduction division of meiosis I does not occur in the asexual females, allowing parthenogenesis. The embryos develop within their asexual mothers and can even contain

*Correspondence: ce75@georgetown.edu

Department of Biology, Georgetown University, 37th and O Streets NW, Washington, DC 20057, USA embryos themselves. Several rapidly developing generations of asexual females are produced until autumn, when the shortened photoperiod induces the last asexual generation to give rise to sexual females and sexual males, completing the cycle. Sex determination in pea aphid is $\mathrm{XX} / \mathrm{XO}$, with males being $\mathrm{XO}$. The males are produced by removal of one $\mathrm{X}$ chromosome during meiosis II. Given that all sperm carry an $\mathrm{X}$ chromosome, the following sexually produced generation is all female [3].

Rapid reproduction during the asexual phase of the life cycle allows aphids to adapt quickly to new environments and host plants, and it has contributed to the development of alternative phenotypes (polyphenisms) among individuals with identical genotypes. These polyphenisms, such as asexual versus sexual females, winged versus wingless asexual females and morphs specialized to resist extreme environments or defend the colony, make the pea aphid a good system for investigating the effect of environmental cues on development [3]. Indeed, Miura et al. [3] found that the development of asexual and sexual embryos was highly divergent, despite being controlled by identical genomes in clonally produced individuals. The pea aphid genome sequence shows remarkably extensive gene duplication, with more than 2,000 gene families that are expanded compared with the published genomes of other insects, suggesting that the unusual developmental patterns may be facilitated by duplications of genes related to development and cell cycle [1]. For example, lineage-specific duplications in several mitotic regulators and mitosis-related genes may contribute to plasticity of the cell cycle [1].

\section{Symbiosis}

In addition to providing a model for phenotypic plasticity, the pea aphid is the best-studied model for maternally transmitted symbionts (reviewed in $[4,5]$ ). Pea aphids have coevolved with the obligate intracellular symbiont Buchnera aphidicola for over 100 million years. Buchnera are Gram-negative bacteria that exist only within specialized cells of pea aphids called bacteriocytes and are transferred vertically from mother to embryos. In addition to the obligate symbiont, pea aphids have more recent associations with vertically transmitted facultative 
symbionts, including the Gram-negative bacteria Regiella insecticola, Serratia symbiotica and Hamiltonella defensa (reviewed in [6]). Although they are not required for host vitality, they confer benefits such as protection against parasitoid wasps, fungal pathogens and heat [6].

Nutritional, physiological and functional studies (reviewed in [5,7]), in addition to a completely sequenced genome of the Buchnera strain that infects the pea aphid [8], have provided clues about the nature of the interdependency between host and symbiont. Annotation of the Buchnera genome [8] supports previous studies indicating that although Buchnera has a dramatically reduced gene repertoire, it provides amino acids that the host cannot produce. The Buchnera genome includes genes involved in biosysnthesis of the nine amino acids that are known to be essential to animals (histidine, isoleucine, leucine, lysine, methionine, phenylalanine, threonine, tryptophan and valine), but very few genes involved in synthesis of non-essential amino acids [8]. Manual annotation of the pea aphid genome indeed shows that it lacks machinery to synthesize the nine amino acids that are essential to other animals [1]. In addition, pea aphid cannot synthesize arginine due to the complete lack of urea cycle genes [1]. Previous studies (for example, [8]) have suggested that the host provides what the symbiont cannot produce. The IAGC [1] confirmed the coordination of metabolism between host and symbiont. For example, rather than excreting nitrogenous waste, pea aphid recycles amino groups as glutamine, which Buchnera then incorporates into the production of arginine $[1,8]$. Remarkably, annotation of the pea aphid genome suggests that several additional amino acid and purine metabolism pathways include steps encoded across the two genomes (see Figure 9 in [1]).

The availability of host and symbiont genomes facilitates the investigation of lateral gene transfer. The previously sequenced genomes of Buchnera (for example, [8]) have shown no evidence of gene uptake from the host [5]. Now, the IAGC has been able to perform the first exhaustive search for lateral gene transfer in the genome of a eukaryotic host that has heritable associations with symbiotic bacteria. They found 12 genes or gene fragments of bacterial origin [1]. Although some of these genes had been found previously to be highly expressed in bacteriocytes so may function in the regulation of the symbiosis [9], overall there was little transfer of bacterial genes to the host genome [1].

\section{Immunity and defense}

Adding to the complexity of the pea aphid system are associations with enemies such as pathogenic fungi and parasitic wasps, which leads to the question of how aphid defense mechanisms operate. Gerardo et al. [2] begin to address that question by manually annotating the pea aphid genome to determine the presence or absence of immune- and stress-related genes found in other insects, such as Drosophila, then performing RNA and protein expression analyses of pathogen-challenged and uninfected aphids. They systematically sought genes related to microbial recognition, signaling pathways and response. Their results show that pea aphids are missing many immune- and stress-related genes found in all other insects with published genomes, and that their RNA and protein expression responses to infection are limited [2].

The most striking differences in microbial recognition genes between pea aphid and other studied insects are the lack of peptidoglycan receptor proteins (PGRPs), class $\mathrm{C}$ scavenger receptors and epidermal growth factor (EGF)-repeat-containing genes in pea aphids [2]. Drosophila PGRPs recognize peptidoglycans in the cell walls of Gram-negative and Gram-positive bacteria, and this leads to the activation of the Toll and immunodeficiency/c-Jun N-terminal kinase (JNK) pathways. The recognition of Gram-positive bacteria in Drosophila is preceded by the formation of a complex between Gramnegative binding proteins (GNBPs) and PGRPs and hydrolysis of peptidoglycans into small fragments by GNBPs. The authors found it surprising that pea aphids have two GNBP paralogs, despite lacking PGRPs, and suggested that GNBPs may have a different role in pea aphids [2]. Pea aphids have no class $C$ scavenger receptors [2], which facilitate phagocytosis in Drosophila. The pea aphid genome also lacks EGF repeats, which are found in members of the Nimrod superfamily, thought to serve as receptors in phagocytosis and bacterial binding in other insects [2].

As for signaling pathways, Gerardo et al. [2] found the Toll and Janus kinase/signal transducer (JAK/STAT) pathways to be intact. These are both thought to be involved in development and innate immunity. On the other hand, they could not identify many components of the immunodeficiency (IMD) signaling pathway, which is critical for fighting Gram-negative bacteria in Drosophila and may also have a role in defense against Gram-positive bacteria and fungi (see Figure 1 in [2]). The IMD pathway genes missing in pea aphid have conserved one-to-one orthologs in most other published insect genome sequences [2]. Since the IMD pathway triggers the JNK pathway in Drosophila, the authors found it surprising that the pea aphid genome does include most components of the JNK pathway [2].

Pea aphids differ extensively in their defense response genes compared with those known in other insects [2]. They are missing many of the antimicrobial peptides (AMPs) that are conserved in other insects (see [2] for a complete list). Notably, pea aphids lack defensins, which have been found in all insect genomes sequenced so far. 
Similar to the red flour beetle (Tribolium castaneum) but unlike any other sequenced insect genome, the pea aphid genome contains plant-like thaumatin homologs, which have anti-fungal properties in plants. The authors [2] suggest that these are ancient defense genes that have been lost in many insect species. Another striking finding is that pea aphid lacks C-type lysozymes, which are the most common class of lysozyme in metazoa and which have been found in all other sequenced insect genomes [2]. Lysozymes are a family of enzymes that degrade bacterial cell walls. Pea aphids do have three i-type (invertebrate) lysozymes [2]. In addition, two genes that were found to be of bacterial origin encode bacteriolytic enzymes similar to lysozymes [1].

Gerardo et al. [2] then went on to investigate expression of 23 of the recognition, signaling and response genes in aphids that had been subjected to infection and stress treatments and, remarkably, found no upregulation of AMPs in infected aphids. Similarly, in expressed sequence tag (EST)-based experiments comparing cDNA libraries synthesized from guts of infected and uninfected aphids, they did not detect any standard immune related genes. They then used suppression subtractive hybridization (SSH) to compare cDNA from infected and uninfected aphids. Briefly, SSH is a technique in which PCR amplification of cDNAs that are common between two samples is selectively suppressed, so that only differentially expressed cDNAs are amplified and subsequently cloned and sequenced. Optimizing the control and experimental sample ratio ensures that cDNAs more abundantly expressed in the experimental sample (in this case infected aphids) are selectively amplified. The infected versus uninfected aphid SSH library included few immune-related genes, and again, no AMPs. Finally, high performance liquid chromatography (HPLC) peptide analyses targeting small peptides, such as AMPs, were run on the hemolymph of infected aphids and also suggested a lack of AMP response [2].

The findings of Gerardo et al. [2] suggest that pea aphids, and possibly other hemimetabolous insects, have a defense system that differs greatly from other wellstudied insects, most of which are holometabolous, bringing the authors to question the generality of the accepted insect model of immunity. Their functional analyses agree with a previous SSH study investigating wound-mediated expression in aphid, which also found no AMPs to be present in hemolymph [10]. Gerardo et al. [2] revisit hypotheses proposed by Altincicek et al. [10] to explain the seemingly deficient antimicrobial defenses in pea aphid and suggest that both increased reproduction following infection and symbiont-mediated host protection may contribute to the aphid's defenses.

In summary, I have highlighted a few of the outcomes of the pea aphid genome analysis, which revealed new perspectives on questions related to aphid phenotypic plasticity, symbiosis and defense mechanisms. As the first genome of a hemimetabolous insect, it will reveal the diversity of biological mechanisms among insects and expand our traditional models of fundamental processes, such as immunity and stress response. Combined with the sequences of several symbiont genomes, the pea aphid genome will advance the study of coevolution and encourage a multi-organismal systems biology approach.

Published: 23 February 2010

\section{References}

1. The International Aphid Genomics Consortium: Genome sequence of the pea aphid Acrythosiphon pisum. PLoS Biol 2010, 8:e1000313.

2. Gerardo NM, Altincicek B, Anselme C, Atamian H, Barribeau SM, de Vos M, Duncan E, Evans JD, Gabaldon T, Ghanim M, Heddi A, Kaloshian I, Latorre A, Moya A, Nakabachi A, Parker BJ, Perez-Brocal V, Pignatelli M, Rhabe Y, Ramsey JS, Spragg C, Tamames J, Tamarit D, Tamborindeguy C, Vincent-Monegat C, Vilcinskas A: Immunity and other defenses in pea aphids, Acyrthosiphon pisum. Genome Biol 2010, 11:R21.

3. Miura T, Braendle C, Shingleton A, Sisk G, Kambhampati S, Stern DL: A comparison of parthenogenetic and sexual embryogenesis of the pea aphid Acyrthosiphon pisum (Hemiptera: Aphidoidea). J Exp Zool B Mol Dev Evol 2003, 295:59-81.

4. Moran NA, McCutcheon JP, Nakabachi A: Genomics and evolution of heritable bacterial symbionts. Annu Rev Genet 2008, 42:165-190.

5. Moran NA, Degnan PH: Functional genomics of Buchnera and the ecology of aphid hosts. Mol Ecol 2006, 15:1251-1261.

6. Oliver KM, Russell JA, Moran NA, Hunter MS: Facultative bacterial symbionts in aphids confer resistance to parasitic wasps. Proc Natl Acad Sci USA 2003, 100:1803-1807.

7. Douglas AE: Nutritional interactions in insect-microbial symbioses: aphids and their symbiotic bacteria Buchnera. Annu Rev Entomol 1998, 43:17-37.

8. Shigenobu S, Watanabe H, Hattori M, Sakaki Y, Ishikawa H: Genome sequence of the endocellular bacterial symbiont of aphids Buchnera sp. APS. Nature 2000, 407:81-86.

9. Nikoh N, Nakabachi A: Aphids acquired symbiotic genes via lateral gene transfer. BMC Bio/ 2009, 7:12.

10. Altincicek B, Gross J, Vilcinskas A: Wounding-mediated gene expression and accelerated viviparous reproduction of the pea aphid Acyrthosiphon pisum. Insect Mol Biol 2008, 17:711-716.

doi:10.1186/gb-2010-11-2-106

Cite this article as: Elsik CG: The pea aphid genome sequence brings theories of insect defense into question. Genome Biology 2010, 11:106. 